\title{
Sporadicity and synchronization in one-dimensional asymmetrically coupled maps
}

\author{
F Cecconi, A Crisanti, M Falcioni $\nmid$ and A Vulpiani $\nmid$ \\ Dipartimento di Fisica, Università di Roma "La Sapienza", P.le A. Moro 2, I-00185 \\ Roma, Italy \\ $\dagger$ INFN, Sezione di Roma
}

Short title: chaos in extended systems

PACS number(s): 05.45.+b

Submitted to: JPA

Date: 4 September 2018 
Abstract. A one-dimensional chain of sporadic maps with asymmetric nearest neighbour couplings is numerically studied. It is shown that in the region of strong asymmetry the system becomes spatially fully synchronized, even in the thermodinamic limit, while the Lyapunov exponent is zero. For weak asymmetry the synchronization is no more complete, and the Lyapunov exponent becomes positive. In addition one has a clear relation between temporal and spatial chaos, i.e.: a positive effective Lyapunov exponent corresponds to a lack of synchronization and vice versa 
An interesting property of extended systems is that they can exhibit complex behaviour both in space and time, that is the chaotic evolution of a spatial pattern. This kind of phenomena, called "spatio-temporal" chaos, has been the focus of considerable interest in different fields, as chemical reaction-diffusion systems, Bénard convection, turbulence, modelling of brain fuction, and so on [1-2]. Recent experiments have revealed the emergence of large-scale spatio-temporal patterns of activity in many brain areas, such as the olfactory system or the visual cortex [3]. Coherent spatial patterns play also an important role in the behaviour of turbulent fluids. Here numerical simulations and experiments show the emergence of strong choerent vortex structures which are responsible for intermittency and possible anomalous dimensions in the scaling law [4]. Moreover choerent structures may play a non trivial role in the predictabilty problem based on two-dimensional turbulence, an important subjec from both theoretical and metereological point of view [5].

A direct study of the spatio-temporal behaviour from the full equations, such as the Navier-Stokes equations, in general, is quite complicated, even numerically. To overcome this problem, and gain more insight in the basic mechanisms, much work has been devoted to the study of models, such as coupled lattice maps. These are a crude, but nontrivial, approximation of extended systems with discrete space and time, but continuous states [2]. The simplest form is given by a set of $N$ continuous variables $x_{i}$ which evolve in (discrete) time as

$$
\begin{aligned}
x_{i}(n+1)= & \left(1-\alpha_{i}-\beta_{i}\right) f\left(x_{i}(n)\right) \\
& +\alpha_{i} f\left(x_{i-1}(n)\right)+\beta_{i} f\left(x_{i+1}(n)\right) .
\end{aligned}
$$

The index $i$ denotes the $i^{t h}$ site on a one-dimensional lattice, and only nearest neighbour interactions are taken into account. The strenght of the couplings and their spatial homogeneity, as well as the type of boundary conditions used, may depend on the the type of physical problem. For example in the case of shear flow, boundary layers or convection, there is a privileged direction. This can be introduced in the model (1) by taking asymmetric couplings $[6,7]$.

Moreover in such systems the most appropriate boundary conditions are open boundary conditions. Thermal reservoir can be included by giving a special evolution law to the sites on the boundary. We do not consider this generalization here.

In this letter we shall study a one-dimensional chain of coupled maps (1) with homogeneous asymmetric couplings and open boundary conditions. The equations of 
motion are given by (1), with couplings

$$
\begin{aligned}
& \alpha_{i}=\gamma_{1}, \quad \beta_{i}=\gamma_{2} \quad \text { for } i=2, \ldots, N-1 \\
& \alpha_{1}=0, \quad \beta_{1}=\gamma_{2}, \quad \alpha_{N}=\gamma_{1}, \quad \beta_{N}=0 .
\end{aligned}
$$

Without loss of generality we assume $\gamma_{1}>\gamma_{2}$. The system (1) with these couplings was studied in Refs. [7,8] for the case of chaotic single map $f(x)$. In these papers the interest was for the possible emergence of spatial coherent patterns due to the asymmetrical couplings.

A simple inspection reveals that the system (1)-(2) posses the uniform solution

$$
x_{i}(n)=\widetilde{x}(n), \quad \widetilde{x}(n+1)=f(\widetilde{x}(n)) .
$$

To study the stability of the uniform state one can linearize eq. (1) about the uniform solution (3) and study the spectrum of the fluctuations [7]. This consists of a uniform eigenmode with eigenvalue $\rho_{0}$ and $N-1$ nonuniform eigenmodes with spectrum

$$
\rho(k)=\rho_{0}\left[1-\gamma_{1}-\gamma_{2}+2 \sqrt{\gamma_{1} \gamma_{2}} \cos (k)\right]
$$

where $k=\pi m / N$ with $m=1,2, \ldots, N-1$, and $\rho_{0}=\exp \left(\lambda_{0}\right)$ where $\lambda_{0}$ is the largest Lyapunov exponent of the single map $f(x)$.

For $\gamma_{1} \neq \gamma_{2}$ this spectrum posses a gap at $k=0$ since $\rho(k \rightarrow 0)$ is less that the $k=0$ eigenvalue $\rho_{0}$. Therefore if $\gamma_{1}$ and $\gamma_{2}$ are such that $\rho_{0}>1$ and $|\rho(k)|<1$ then all nonuniform fluctuations are stable. The only instability that is left is the instability to uniform fluctuations, which is inherent to the chaotic nature of the single map.

¿From this one concludes that the uniform state (3) is stable. This predictions for the case of a single chaotic map $f(x)$ are essentially confirmed by numerical simulations $[7,8]$. The finite coherence length $l_{\mathrm{c}}-$ that is: $x_{i}(n) \simeq \widetilde{x}(n)$ for $i<l_{\mathrm{c}}$, while for $i>l_{\mathrm{c}}$ the $x_{i}$ are spatially irregular - is due to numerical noise. Indeed $l_{\mathrm{c}}$ decreases logarithmically with the noise level in the numerical simulations.

The open boundary condition can be seen as a defect in the chain. This scenario is essentially unchanged if one uses periodic boundary conditions and "softer" defects, such as, e.g., the interchange of $\gamma_{1}$ and $\gamma_{2}$ in a finite fraction of sites [8].

The above argument neglects the fluctuations of the chaotic degree along the trajectory, since the Lyapunov exponent $\lambda_{0}$ gives only the typical chaotic degree. If one considers a temporal window $[t-\Delta t / 2, t+\Delta t / 2]$ it is possible to repeat the 
arguments of Ref. [7] simply replacing $\lambda_{0}$ in equation (4) with the effective Lyapunov exponent [9] which measures the local exponential rate of growth for the tangent vector $\mathbf{z}$ around the time $t$ :

$$
\chi_{\Delta t}(t)=\frac{1}{\Delta t} \ln \frac{|\mathbf{z}(t+\Delta t / 2)|}{|\mathbf{z}(t-\Delta t / 2)|}
$$

We expect that the scenario discussed in Ref. [7] may fail if the fluctuations of $\chi_{\Delta t}$ are strong. It is hence interesting to inquire what happens in the extreme cases.

A rather natural way to address this problem is to study the system (1)-(2) with the single map

$$
f(x)=x+c x^{z} \quad \bmod 1 \quad(z \geq 1) .
$$

For $z \geq 2$ the dynamical system $x(n+1)=f(x(n))$ shows a sporadic behaviour [10], i.e. an initial disturbance grows in time as a stretched exponential with exponent less that one. The Lypunov exponent $\lambda_{0}$ hence vanishes.

In the case of $1 \leq z<2$ the map (6) behaves as an ordinary chaotic system with positive Lyapunov exponent $\lambda_{0}$. Here we obtain for the system (1) the scenario discussed in Refs. $[7,8]$ for the logistic map. We thus find that for symmetric couplings the chain does not synchronize, while in the asymmetric case a finite coherence length may appear depending on the value of $\gamma_{1}-\gamma_{2}$ and $\lambda_{0}$. To be more specific we can distinguish two cases. In the first case $\rho_{0}>1$ and $|\rho(k)|<1-e . g$. when $z=1.5$, for which $\lambda_{0}=0.56, \gamma_{1}=0.7$ and $\gamma_{2}=0.01$ - so that all the non-uniform eigenmodes are stable an the system synchronizes with a finite $l_{\mathrm{c}}[7,8]$. In the second case - e.g. when $z=1.5, \gamma_{1}=0.7$ and $\gamma_{2}=0.1$ - there exist some $k$ for which $|\rho(k)|>1$, and the system does not synchronize.

We now turn to the case of $z \geq 2$, when the single map is sporadic. The results we shall report are obtained from numerical simulations with $\gamma_{1}=0.7$ and $\gamma_{2}$ which varies in the range $\left(0, \gamma_{1}\right]$. The typical sistem size considered is $N=200$, but we checked some results also for larger values of $N$.

If the couplings are symmetric the systems is not spatially synchronized, $l_{\mathrm{c}} \simeq 1$. Moreover, even if the Lyapunov exponent of the single map $\lambda_{0}$ is zero, the Lyapunov exponent $\lambda$ of the global system is always positive confirming the non relevance of the uniform mode for the dynamics.

As for the case of a chaotic map $f(x)$, when the couplings are not equal two qualitative different behaviours appear depending on the value of $\gamma_{1}-\gamma_{2}$. However, 
unlike the previous case, here the synchronization, when present, is complete, i.e. $l_{\mathrm{c}}=N$. In Fig.1 we show $\tau$ - the fraction of the time the system is completely synchronized - and $\lambda$ - the Lyapunov exponent - as functions of $\gamma_{2}$.

A first regime, with $\tau \simeq 1$, appears for $\gamma_{1}-\gamma_{2}$ large, which with our parameters means $\gamma_{2} \leq 0.2$. Here $\rho_{0}=1$ and $|\rho(k)|$ is well smaller than one for all $k$, e.g., $\max _{k}|\rho(k)|=0.85$ for $\gamma_{2}=0.2$. According to the above argument the uniform solution is locally stable. Indeed we find that the system synchronizes with $l_{\mathrm{c}}=N$. A detailed analysis of the evolution reveals that the system spends most of the time completely synchronized. The ordered state is interrupted by intervals where the coherence length becomes very small. This is a very different qualitative behaviour with respect to the case $z<2$ and the case discussed in Refs. [7,8]. In fact in these latter cases the system is always partially synchronized, with $l_{\mathrm{c}}$ undergoing small fluctuations about its time average. All the simulations in this range of parameters gives a value of $\tau$ very close to one. For example, when $\gamma_{2}=0.1$ we find, on a run of $5 \times 10^{6}$ steps, $\tau=0.997$, while for $\gamma_{2}=0.2$, and on a run of $4.7 \times 10^{8}$ steps, we find $\tau=0.999$. The Lyapunov exponent is always very small. It is difficult to decide if $\lambda$ is really going to zero or stays very small. This difficulty is due to the fact that every time the system desynchronizes, the local Lyapunov exponent increases. As a consequence the numerical value of $\lambda$ does not saturate in time but has kicks which increase its value, see Fig.2. We note that this incertitude on the value of $\lambda$ is directly connected to that on $\tau$.

A different behaviour appears for $\gamma_{1}-\gamma_{2}$ small which, with our parameters, means $\gamma_{2} \geq 0.35$. In this regime $\rho_{0}=1$ but $|\rho(k)|$ is larger than one for some values of $k$, e.g., when $\gamma_{2}=0.35$ we have $\max _{k}|\rho(k)|=1.04$. Thus the uniform state is locally unstable. Indeed we find that the coherence length is almost always $l_{\mathrm{c}} \simeq 1$ and the system is never completely synchronized - at least on a run of $3 \times 10^{7}$ steps. Moreover $\tau$ is zero and $\lambda$ is positive. For example, $\lambda=0.74$ when $\gamma_{2}=0.35$.

These two behaviours appear to be related in a continuous way through the crossover region where $\max _{k}|\rho(k)|$ is very close to one, for example $\max _{k}|\rho(k)|=0.92$ for $\gamma_{2}=0.3$. In this region $\lambda$ begins to be definitely non zero and $\tau$ becomes smaller than one. For example, $\lambda \simeq 0.05$ and $\tau \simeq 0.9$ again for $\gamma_{2}=0.3$ on a run of $3 \times 10^{8}$ time steps.

In order to stress the correlation between desynchronization and temporal 
chaoticity, we show in Fig.3 the effective Lyapunov exponent $\chi_{\Delta t}(t)$ and $l_{\mathrm{c}}(t)$ as functions of $t$. It is evident that a large $\chi_{\Delta t}(t)$ corresponds to $l_{\mathrm{c}}<<N$ and viceversa. This happens both for small and large values of $\gamma_{1}-\gamma_{2}$.

We stress that since the map $f(x)$ is sporadic, and hence does not posses an asymptotic measure, the initial condition is relevant. In fact while the qualitative behaviour described above does non change, at least for the values of $\gamma_{2}$ we used, the numerical value of $\lambda$ and $\tau$ my depend on the chosen initial condition.

This dependence is more dramatic in the crossover region, and makes difficult to define clear boundaries.

We conclude with some remarks. The behaviours we observed in the sporadic case are rather different from those one previously studied $[7,8]$ :

a) one has a complete synchronization, i.e. $l_{\mathrm{c}}=N$;

b) this complete synchronization does not change by the addition of a small noise, the effect of the noise is a small decreasing of the fraction, $\tau$, of the time in which $l_{\mathrm{c}}=N$; c) there is a strong correlation between the spatial and temporal behaviour, in particular one observes that the effective Lyapunov exponent $\chi_{\Delta t}(t)$ is large in the intervals of time in which $l_{\mathrm{c}}(t)<<N$, while $\chi_{\Delta t}(t) \simeq 0$ if $l_{\mathrm{c}}(t)=N$. 


\section{References}

[1] Kuramoto Y. 1984 Chemical Oscillations, Waves and Turbulence (SpringerVerlag, New-York)

[2] Crutchfield J P and Kaneko K 1987 Directions in chaos ed. Hao B L (Singapore: World Scientific) p 272

Kaneko K 1990 Formation, Dynamics and Statistics of Patterns ed.s Kawasaky K, Onnky A and Suzuki M (Singapore: World Scientific)

[3] Gray C M, Konig P, Engel A K and Singer W 1989 Nature 338334 Eckhorn R et al. 1988 Biol. Cybern. 60121

Skarda C A and Freeman W J 1987 Behaviour. Brain Sci. 10161

[4] Benzi R, Paladin G, Patarnello S, Santangelo P and Vulpiani A 1986 J. Phys. A 193771

Carnevale G F, McWilliams J C, Pomeau Y, Weiss J B and Young W R 1991 Phys. Rev. Lett. 662735

[5] Leith C E 1971 J. Atmos. Sci. 28145

[6] Jensen M H 1989 Phys. Rev. Lett. 621361

Jensen M H 1989 Physica D 32203

[7] Aranson I, Golomb D and Sompolinsky H 1992 Phys. Rev. Lett. 683494

[8] Biferale L, Crisanti A, Falcioni M and Vulpiani A 1993 J. Phys. A 26 L923

[9] Paladin G and Vulpiani A 1987 Phys. Rep. 156147

[10] Gaspard P and Wang X-J 1988 Proc. Natl. Acad. Sci. USA 854591 


\section{Figure captions}

Figure 1. Lyapunov exponent $\lambda$ (crosses) and probability of complete synchronization $\tau$ (squares) vs $\gamma_{2} . \quad N=200$ and $\gamma_{1}=0.7$. The integration steps are $3 \cdot 10^{8}$ for $\gamma_{2}=0.3,4.7 \cdot 10^{8}$ for $\gamma_{2}=0.2$ and a few millions of steps in the other cases.

Figure 2. Time evolution of the mean value defining the Lyapunov exponent $\lambda$, for $\gamma_{2}=0.2$ (lower curve) and $\gamma_{2}=0.3$.

Figure 3. Effective Lyapunov exponent $\chi_{\Delta t}$ and coherence length $l_{\mathrm{c}}$ vs $t . N=200$, $\gamma_{1}=0.7, \gamma_{2}=0.3, \Delta t=3 \cdot 10^{4}$. 


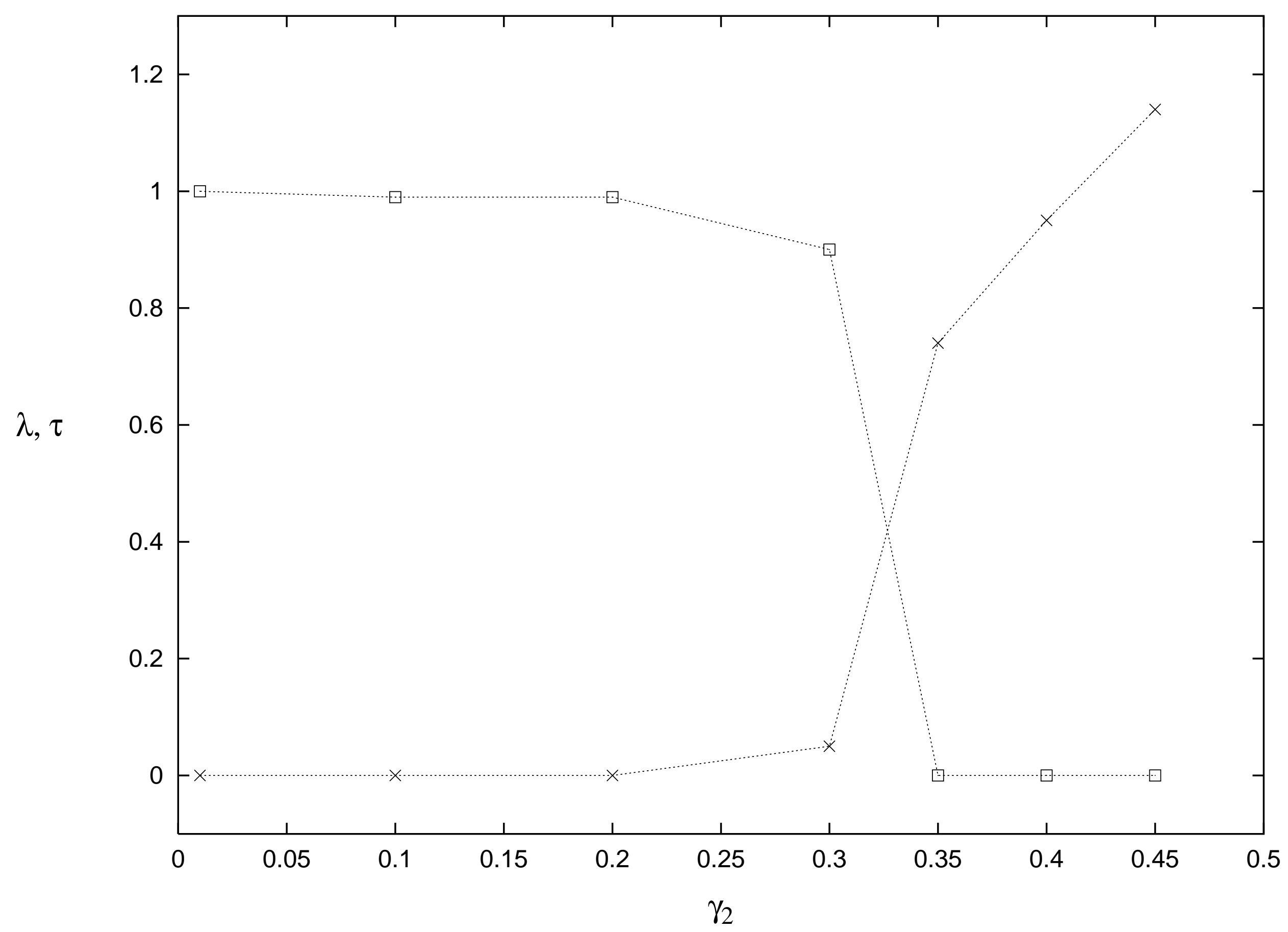




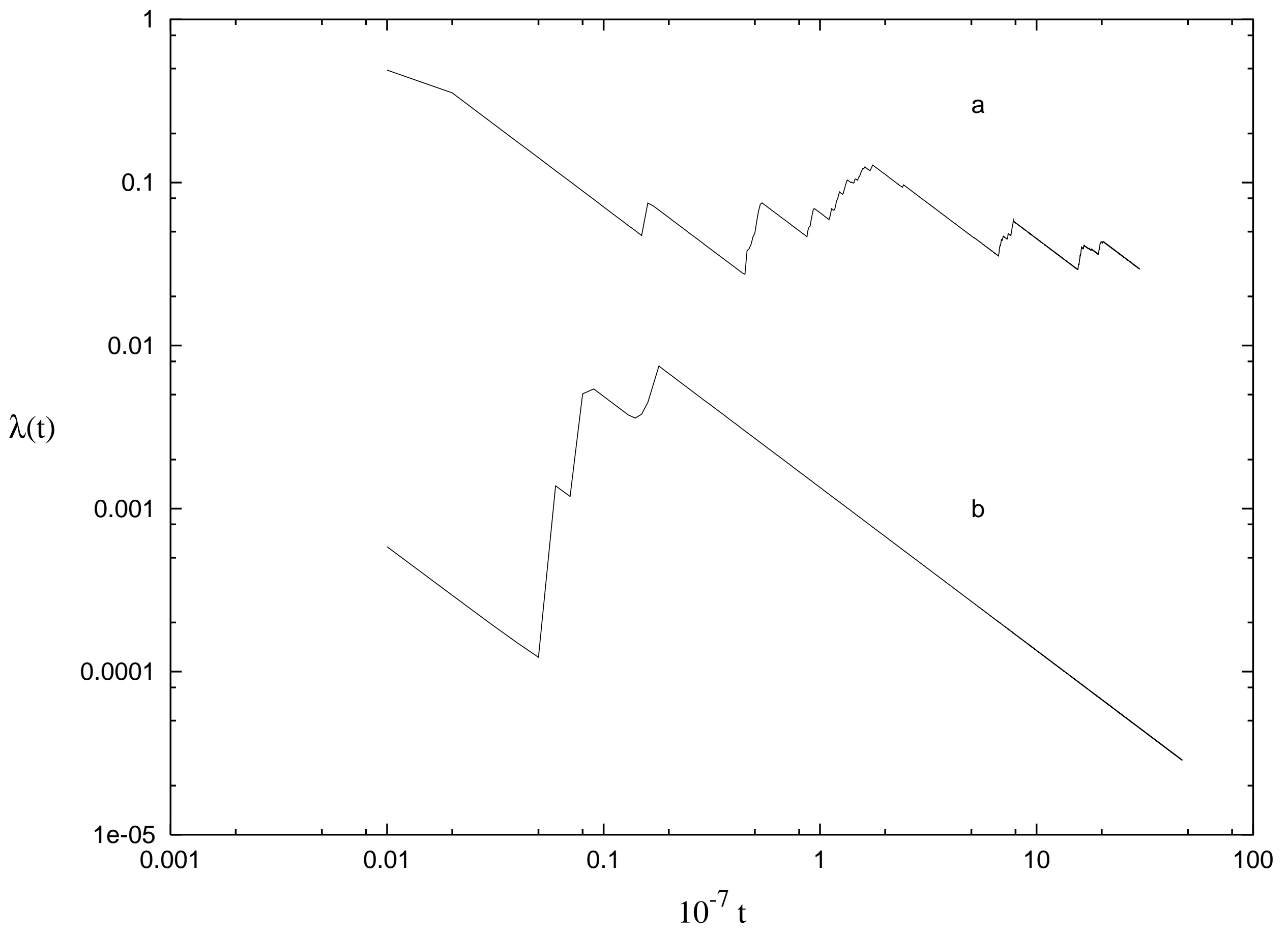




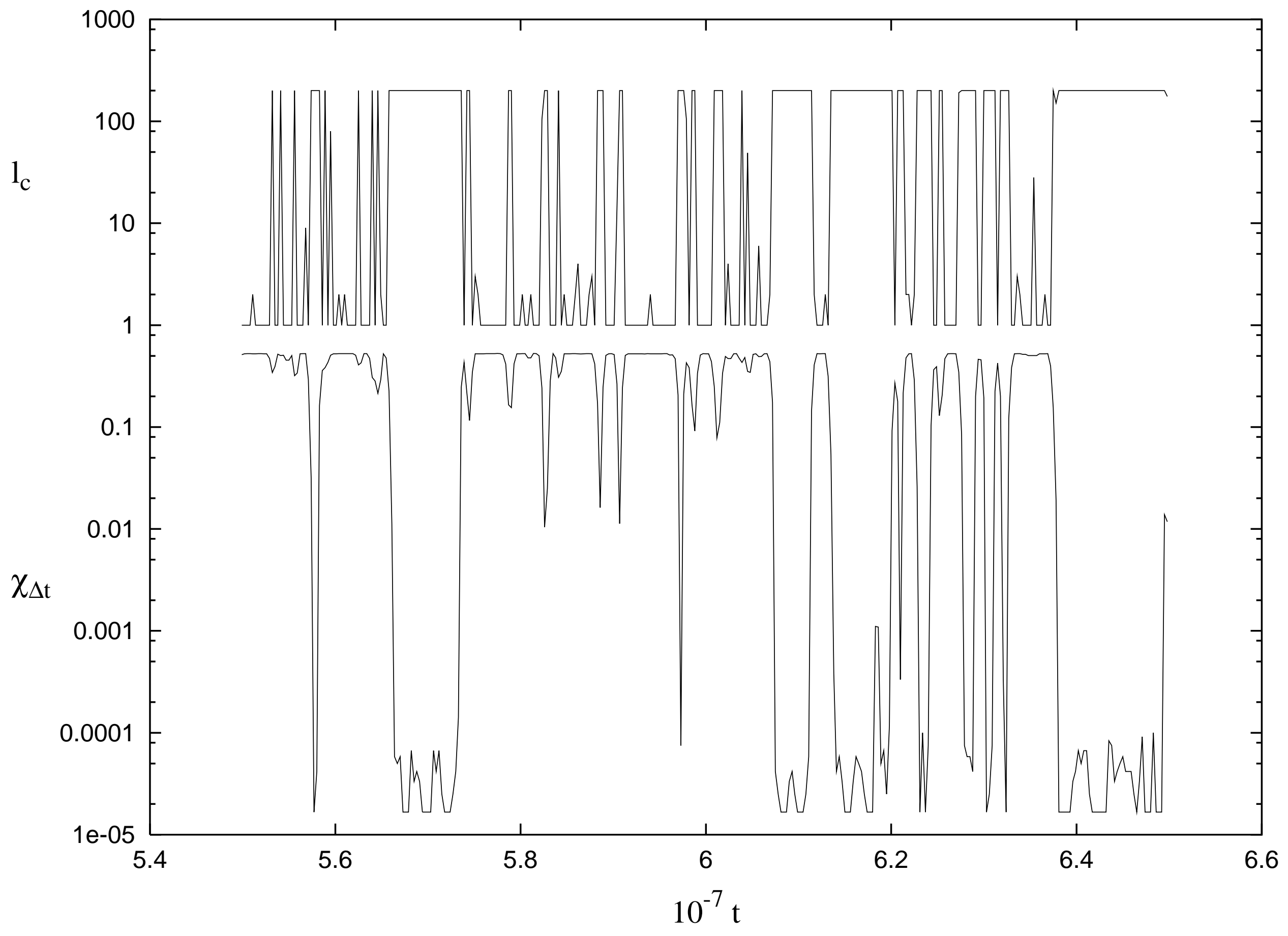

